\title{
Daniel H. Kim, Randal R. Betz, Stephen L. Huhn, Peter O. Newton: Surgery of the pediatric spine
}

\author{
Georg Thieme Verlag, Stuttgart, New York, 2007, 928 pp., 1050 figs. 22 cm, \\ Hardcover EUR (D) 259,95 CHF 432,00, ISBN 13: 978-3 13 141931-6
}

\author{
Pierre H. Kehr • Claude Karger
}

Received: 11 June 2009 / Accepted: 30 June 2009 / Published online: 30 July 2009

(C) Springer-Verlag 2009

\begin{abstract}
Aimed towards both pediatric neurosurgeons and orthopedic surgeons, this 876 pages textbook is a multidisciplinary reference for the surgical management of the most frequently encountered spine problems in children. In the past, most of the available sources of information regarding pediatric spine surgery were embedded in multiple manuals. This book represents one of the first volumes entirely and specifically devoted to pediatric spine surgery, written by the leaders in orthopedic and neurological care of spine disorders in children.
\end{abstract}

The book is divided into 11 chapters.

An overview of developmental and clinical aspects provides essential information on biomechanics, neuroimaging, preoperative evaluation, anesthesia, and neurophysiological monitoring. Surgical anatomy and various approaches to the spine and spinal cord are presented with numerous outstanding illustrations. All the chapters are grouped into easy-to-reference sections that are organized by the type of problem, including congenital anomalies and developmental disorders: neoplasms, vascular malformations, inflammatory and infectious diseases, neuromuscular disease, trauma, and deformities. The book also presents special techniques for the treatment of spinal deformities, such as osteotomy, vertebrectomy, VEPTR, expansion thoracoplasty, and fusion less techniques. The final chapter is devoted to the rehabilitation of children with spinal cord injuries and covers the principles and key concepts in the treatment, as well as the possible secondary complications and challenges that are unique to pediatric patients.

This book can be considered as an invaluable resource for multidisciplinary approaches to patient care. It provides readers with a solid foundation in the specific issues associated with treating the pediatric patient with spine diseases and disorders, from a combined neurosurgical and orthopedic point of view.

Conflict of interest statement No funds were received in support of this study.

P. H. Kehr $(\square) \cdot$ C. Karger

SOTEST, Strasbourg, France

e-mail: kehrpier@aol.com 